\title{
Self-organized nanogratings in glass irradiated by ultrashort light pulses
}

\section{$\operatorname{AUTHOR}(\mathrm{S})$ :}

Shimotsuma, Y; Kazansky, PG; Qiu, JR; Hirao, K

\section{CITATION:}

Shimotsuma, Y ... [et al]. Self-organized nanogratings in glass irradiated by ultrashort light pulses. Physical Review Letters 2003, 91(24): 247405.

\section{ISSUE DATE:}

2003-12-12

URL:

http://hdl.handle.net/2433/39921

RIGHT:

Copyright 2003 American Physical Society 


\title{
Self-Organized Nanogratings in Glass Irradiated by Ultrashort Light Pulses
}

\author{
Yasuhiko Shimotsuma \\ R\&D Center Kagoshima, Kyocera Corporation, Kagoshima, Kokubu 899-4312, Japan \\ Peter G. Kazansky \\ Photon Craft Project, Japan Science and Technology Corporation, Keihanna-Plaza, Kyoto 619-0237, Japan \\ and Optoelectronics Research Centre, University of Southampton, SO17 1BJ, United Kingdom \\ Jiarong Qiu \\ Photon Craft Project, Shanghai Institute of Optics and Fine Mechanics, Chinese Academy of Sciences and Japan Science and \\ Technology Corporation, Keihanna-Plaza, Kyoto 619-0237, Japan \\ Kazuoki Hirao \\ Department of Material Chemistry, Graduate School of Engineering, Kyoto University, Kyoto, Sakyo-ku 606-8501, Japan
}

(Received 21 March 2003; published 11 December 2003)

\begin{abstract}
Periodic nanostructures are observed inside silica glass after irradiation by a focused beam of a femtosecond Ti:sapphire laser. Backscattering electron images of the irradiated spot reveal a periodic structure of stripelike regions of $\sim 20 \mathrm{~nm}$ width with a low oxygen concentration, which are aligned perpendicular to the laser polarization direction. These are the smallest embedded structures ever created by light. The period of self-organized grating structures can be controlled from $\sim 140$ to $320 \mathrm{~nm}$ by the pulse energy and the number of irradiated pulses. The phenomenon is interpreted in terms of interference between the incident light field and the electric field of the bulk electron plasma wave, resulting in the periodic modulation of electron plasma concentration and the structural changes in glass.
\end{abstract}

DOI: 10.1103/PhysRevLett.91.247405

Progress in high power ultrashort pulse lasers has opened new frontiers in physics and technology of light-matter interactions. Recent discoveries span from coherent $\mathrm{x}$-ray generation [1,2] and nonlinear Thomson scattering [3] to direct writing of 3D photonic structures [4-8]. A critical advantage of using femtosecond pulses relative to longer pulses for direct writing and data storage is that such pulses can rapidly and precisely deposit energy in solids $[9,10]$. Although molecular defects caused by such intense irradiation have been identified in fluorescence, ESR, and other studies [11,12], the mechanism of induced modifications in glass is still not fully understood. Moreover, such structures in Ge-doped silica [4] and other glass materials [13] show an unexpected anisotropic light scattering which peaks in the plane of light polarization. This has been interpreted in terms of photoelectrons moving along the direction of light polarization inducing subwavelength size stripelike index inhomogeneities. Another puzzling phenomenon is uniaxial birefringence imprinted in structures written within fused silica plates [14]. The observation of a strong reflection from the modified regions occurring only along the direction of the polarization of the writing laser has given the evidence of periodic, subwavelength gratinglike distribution of the index inhomogeneities in the irradiated spot inside the glass, which could also explain the birefringence phenomenon [15]. Surface ripples with a period equal to the wavelength of incident laser radiation and that are likewise aligned in a direction perpendicular to
PACS numbers: 78.20.Nv, 42.65.Sf, 42.79.Dj, 79.20.Ds

the electric field of light wave have been observed in many experiments involving laser deposition [16] and laser ablation [17]. Such gratings are generated as a result of interference between the light field and the surface plasmon-polariton wave launched because of initial random surface inhomogeneities. However, until now there has been no observation of periodic structures being generated within the bulk of a material just by a single writing laser beam and the mechanism of its appearance has not been fully understood. Here we report the first observation of the emergence of periodic structurally changed regions of nanometer size inside silica glass after irradiation by intense femtosecond light pulses. The phenomenon is interpreted in terms of interference between the incident light field and the electric field of bulk electron plasma wave, resulting in periodic modulation of electron plasma concentration and permanent structural changes in glass.

In our experiments, we used commercially available synthetic silica glass, ED-H (Tosoh Quartz Corp., brands of wet silica with $\mathrm{OH} \sim 50 \mathrm{ppm}$ ) of $10 \mathrm{~mm} \times 10 \mathrm{~mm} \times$ $2 \mathrm{~mm}$ size. The laser radiation in Gaussian mode produced by regenerative amplified mode-locked Ti:sapphire laser (150 fs pulse duration, $200 \mathrm{kHz}$ repetition rate) operating at a wavelength of $800 \mathrm{~nm}$ was focused via $100 \times$ (numerical aperture $=0.95$ ) microscope objective into the silica glass samples placed on the $X Y Z$ piezotranslation stage. The beam was focused at $\sim 100 \mu \mathrm{m}$ below the surface and the beam waist diameter was 
estimated to be $\sim 1 \mu \mathrm{m}$. The laser writing parameters were controlled by an electronic shutter, a variable neutral density filter, and a half-wave plate placed in an optical path of the laser beam.

After laser irradiation, the sample was polished to the depth of the beam waist location. The surface of the polished sample was analyzed by scanning electron microscope (JEOL, model JSM-6700F) and Auger electron spectroscopy (PHI, model SAM-680). Secondary electron (SE) images and backscattering electron (BE) images of the same surface were compared (Fig. 1). It is well known that the SE image reveals the surface morphology of a sample, while the BE image is sensitive to the atomic weight of the elements or the density of material constituting the observation surface. The SE images of the polished silica sample indicate that the morphology of an irradiated sample in the examined cross section almost does not change, namely, a void does not exist. On the other hand, the BE images reveal a periodic structure of stripelike dark regions with low density of material and of $\sim 20 \mathrm{~nm}$ width which are aligned perpendicular to the writing laser polarization direction. Horizontal striation, slightly visible in the SE images [Fig. 1(a)], could be explained by a weak surface relief created in the polishing process due to the density variations in the grating. We speculated, based on the fact that the elements constituting the sample are silicon and oxygen (average molecular weight of $\mathrm{SiO}_{2}$ glass $\sim 60.1$ ), that the oxygen defects were formed in the regions corresponding to dark domains of the $\mathrm{BE}$ image, which reduce the average molecular weight in these regions $\left(\mathrm{SiO}_{2-x} \sim 60.1-16 x\right)$. To test this sug-
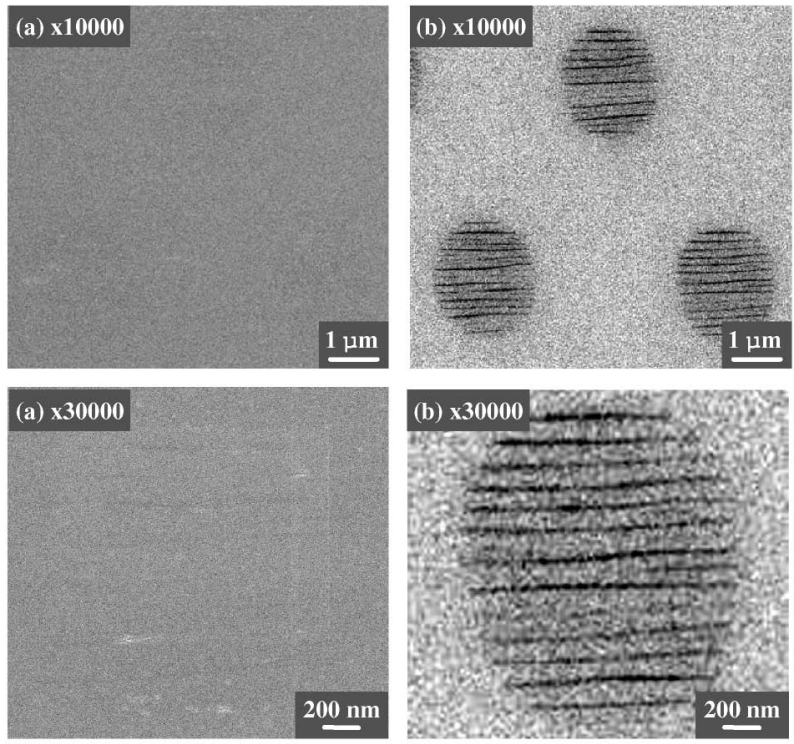

FIG. 1. (a) Secondary electron images of silica glass surface polished close to the depth of focal spot. (b) Light "fingerprints": Backscattering electron images of the same surface. The magnification of the upper and lower images is $10000 \times$ and $30000 \times$, respectively. gestion, we carried out Auger spectra mapping of silicon and oxygen on the same surface with $10 \mathrm{~nm}$ spatial resolution (Fig. 2). The Auger signal of the oxygen in the regions corresponding to dark domains in the $\mathrm{BE}$ image is lower compared to other regions, indicating low oxygen concentration in these domains [Fig. 2(a)]. Furthermore, the intensity of the oxygen signal is stronger in the regions between the dark domains of the BE image. On the other hand, the intensity of the silicon signal is the same in the whole imaged region [Fig. 2(b)]. These results indicate that the periodic structure observed in the BE image consists of periodically distributed oxygen-deficient regions $\left(\mathrm{SiO}_{2-x}\right)$. The Auger signal intensity is proportional to the concentration of element constituting the surface, which gives an estimate to the value $x \sim 0.4$.

We observed the decrease of the grating period with an increase of the exposure time. The grating periods were about 240,180 , and $140 \mathrm{~nm}$ for the number of light pulses of $5 \times 10^{4}, 20 \times 10^{4}$, and $80 \times 10^{4}$, respectively, and for the pulse energy of $1 \mu \mathrm{J}$, corresponding to intensity of $2 \times 10^{14} \mathrm{~W} / \mathrm{cm}^{2}$. This indicated a logarithmic dependence of the grating period $\Lambda$ on the number of light pulses.

The dependence of the observed periodic nanostructures on pulse energy for a fixed exposure time was also investigated and an increase of the period with the pulse energy was observed. Grating periods of 180, 240, and $320 \mathrm{~nm}$ were measured at pulse energies of 1,2 , and $2.8 \mu \mathrm{J}$, respectively, and for the number of light pulses of $20 \times 10^{4}$ (Fig. 3).
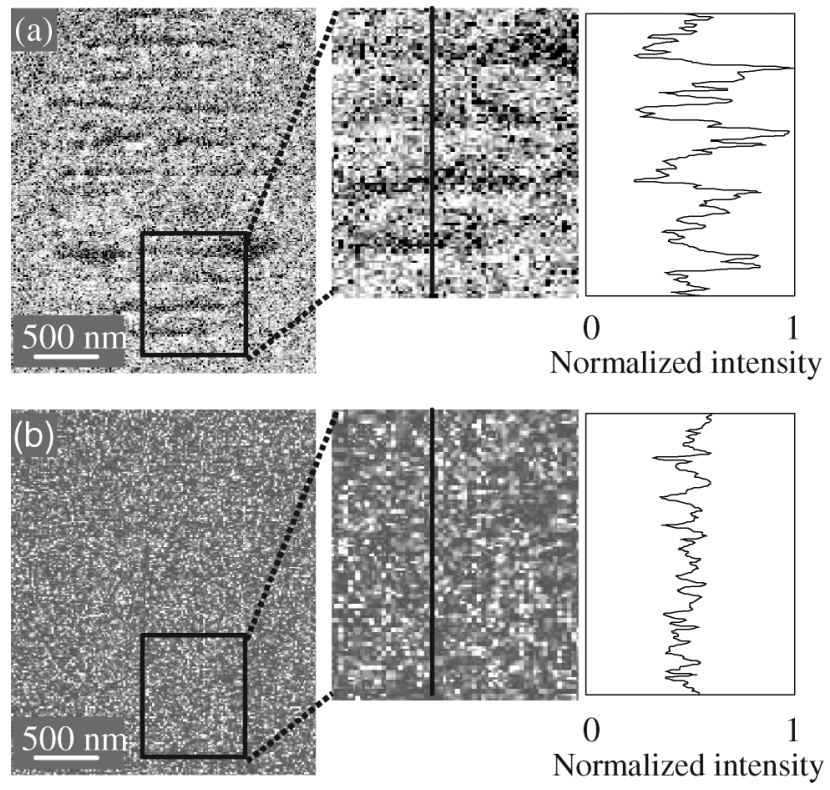

FIG. 2. Auger spectra maps and corresponding line scans of oxygen (a) and silicon (b) on same silica glass surface polished close to the depth of focal spot. 
The following explanation of the observed phenomenon is proposed. Once a high free electron density is produced by multiphoton ionization, the material has the properties of plasma and will absorb the laser energy via one-photon absorption mechanism of inverse bremsstrahlung (joule) heating. The light absorption in the electron plasma will excite bulk electron plasma density waves. These are longitudinal waves with the electric field component parallel to the direction of propagation. Such an electron plasma wave could couple with the incident light wave only if it propagates in the plane of light polarization. Initial coupling is produced by inhomogeneities induced by electrons moving in the plane of light polarization [4]. The coupling is increased by a periodic structure created via a pattern of interference between the incident light field and the electric field of the bulk electron plasma wave, resulting in the periodic modulation of the electron plasma concentration and the structural changes in glass. A positive gain coefficient for the plasma wave will lead to an exponential growth of the periodic structures oriented perpendicular to the light polarization, which become frozen within the material. The electron plasma wave is efficiently generated only with wave vector $k_{\mathrm{pl}}\left(k_{\mathrm{pl}}=\omega_{\mathrm{pl}} / v_{\mathrm{pl}}\right.$, where $v_{\mathrm{pl}}$ is the speed and $\omega_{\mathrm{pl}}$ is the angular frequency of the plasma wave) in the plane of light polarization and only in the direction defined by conservation of longitudinal component of the momentum (Fig. 3 inset). The latter condition is similar to the condition in Cherenkov's mechanism of nonlinear wave generation [18]. The period of the grating

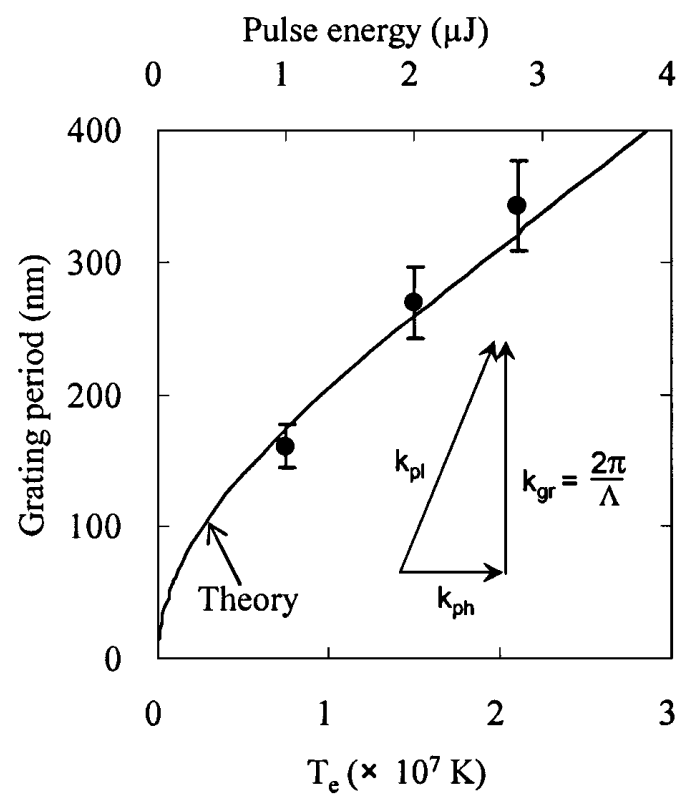

FIG. 3. Theoretical dependence of self-organized grating period on electron temperature for $n_{e}=1.7 \times 10^{21} \mathrm{~cm}^{-3}$. Note that this is not a fit of experimental data. Experimental grating periods versus pulse energy are also shown. Inset is a wave vector matching diagram. is defined by this momentum conservation condition: $k_{\mathrm{gr}}=2 \pi / \Lambda=\sqrt{k_{\mathrm{pl}}^{2}-k_{\mathrm{ph}}^{2}}$, where $k_{\mathrm{ph}}=\omega n / c$ is the wave vector, $\omega$ is the angular frequency, $n$ is the refractive index, and $c$ is speed of light. This relation can also be used to estimate the value of electron plasma wave vector $k_{\mathrm{pl}}=\sqrt{k_{\mathrm{ph}}^{2}+(2 \pi / \Lambda)^{2}}$ which gives $k_{\mathrm{pl}}=43.5 \times$ $10^{4} \mathrm{~cm}^{-1}$, assuming $\omega=2.36 \times 10^{15} \mathrm{~s}^{-1}$ and $\Lambda=$ $150 \mathrm{~nm}$. The dispersion relation for the electron plasma waves or Langmuir waves is as follows: $\omega_{\mathrm{pl}}^{2}=\omega_{p}^{2}+$ $\frac{3}{2} v_{e}^{2} k_{\mathrm{pl}}^{2}$, where $\omega_{p}=\sqrt{\left(N_{e} e^{2}\right) /\left(\varepsilon_{0} m_{e}\right)}$ is the plasma frequency, $N_{e}$ is the electron density, $m_{e}$ is the electron mass, $e$ is the electron charge, $v_{e}=\sqrt{2 k_{B} T_{e} / m_{e}}$ is the thermal speed of electrons, $T_{e}$ is the electron temperature, and $k_{B}$ is Boltzmann constant. Taking into account the energy conservation condition $\omega_{\mathrm{pl}}=\omega$, the momentum conservation relation, and the above dispersion relation, it is possible to obtain an analytical expression for the grating period versus electron temperature and density:

$$
\Lambda=\frac{2 \pi}{\sqrt{\frac{1}{T_{e}}\left(\frac{m_{e} \omega^{2}}{3 k_{B}}-\frac{e^{2} N_{e}}{3 \varepsilon_{0} k_{B}}\right)-k_{\mathrm{ph}}^{2}}} .
$$

This dependence shows that the grating period increases with the increase of electron concentration and electron temperature (Fig. 3). $\Lambda$ increases steeply when the electron concentration approaches $N_{e}^{\mathrm{cr}}-3 \varepsilon_{0} k_{B} k_{\mathrm{ph}}^{2} T_{e} e^{-2} \approx$ $N_{e}^{\mathrm{cr}}$, where $N_{e}^{\mathrm{cr}}$ is critical plasma density $\left[\omega_{p}\left(N_{e}^{\mathrm{cr}}\right)=\omega\right.$, $\left.N_{e}^{\mathrm{cr}}=1.75 \times 10^{21} \mathrm{~cm}^{-3}, \lambda_{\omega}=800 \mathrm{~nm}\right]$. It also follows from above that for a given grating period the electron temperature linearly decreases with increase of the electron concentration:

$T_{e}=A(\Lambda)-B(\Lambda) N_{e}$, where $A(\Lambda)=\left(m_{e} \omega^{2}\right) /\left(3 k_{B} k_{\mathrm{pl}}\right)$, $B(\Lambda)=e^{2} /\left(3 \varepsilon_{0} k_{B} k_{\mathrm{pl}}\right)$, and $A(\Lambda) / B(\Lambda)=N_{e}^{\mathrm{cr}}$. This gives a wide range of realistic [6,9] electron temperatures (e.g., up to $T_{e}=\left(m_{e} \omega^{2}\right) /\left(3 k_{B} k_{\mathrm{pl}}\right)=5 \times 10^{7} \mathrm{~K}$ for $\Lambda=$ $150 \mathrm{~nm}$ ) and densities (up to $1.75 \times 10^{21} \mathrm{~cm}^{-3}$ ) which could explain a certain grating period, including the periods observed in our experiments. For comparison of the theory and experiment, it is useful to define the dependence of the grating period on a measurable value, such as pulse energy. Then, the dependences of electron temperature and density on light intensity should be known. These dependences are complicated and are not very well established in bulk silica $[9,19]$. However, it is certain that the electron temperature will increase and density will not decrease with the light intensity. Then it follows from (1) that the grating period will increase with the pulse energy. The observed increase of the grating period with the pulse energy is in agreement with this theoretical prediction.

Based on the above mechanism of grating formation, it is possible to give the following explanation of the observed formation of stripelike regions with low oxygen concentration. The plasma electrons are created in the 
process of breaking of Si-O-Si bonds via multiphoton absorption of light which is accompanied by the generation of a Si-Si bonds, nonbridging oxygen-hole centers $\left(\mathrm{NBOHC}, \equiv \mathrm{Si}-\mathrm{O}^{-}\right)$and interstitial oxygen atoms $\left(\mathrm{O}_{i}\right)$. Such oxygen atoms are mobile and can diffuse from the regions of high concentration. Negatively charged oxygen ions can be also repelled from the regions of high electron concentration. The photoluminescence and electron spin resonance spectra confirmed the presence of nonbridging oxygen defects and $E^{\prime}$ centers in the irradiated samples. The small thickness of these regions, compared to the period of the grating, could be explained by a highly nonlinear dependence of the structural changes on the electron concentration. Major changes in composition take place after the attainment of thermal equilibrium, involving formation and decay of defect states, such as oxygen vacancies [20]. A detailed mechanism of the structural changes responsible for the nanograting formation is under investigation.

Another puzzling observation, which needs further investigation, is the decrease of the grating period with the number of writing pulses. One possible explanation is that the accumulated structural changes in glass during irradiation result in the reduction of the electron plasma concentration. As follows from (1), a small reduction in the electron density in the vicinity of critical plasma density could lead to a significant reduction of the grating period.

Apart from the fundamental importance of the observed phenomenon as the first direct evidence of interference between light and electron density waves, the observed light "fingerprints" are the smallest embedded structures ever created by light, which could be useful for optical recording and photonic crystal fabrication.

We would like to thank M. Tanaka, A. Fukuura, T. Kishino, M. Nishimura, and K. Furusawa for helpful discussions.

[1] Z. Chang, A. Rundquist, H. Wang, M. M. Murnane, and H. Kapteyn, Phys. Rev. Lett. 79, 2967 (1997).
[2] Ch. Spielmann, N. H. Burnett, S. Sartania, R. Koppitsch, M. Schnurer, C. Kan, M. Lenzner, P. Wobrauschek, and F. Krausz, Science 278, 661 (1997).

[3] S. Chen, A. Maksimchuk, and D. Umstadter, Nature (London) 396, 653 (1998).

[4] P. G. Kazansky, H. Inouye, T. Mitsuyu, K. Miura, J. Qiu, K. Hirao, and F. Starrost, Phys. Rev. Lett. 82, 2199 (1999).

[5] K. Miura, J. Qiu, H. Inouye, T. Mitsuyu, and K. Hirao, Appl. Phys. Lett. 71, 3329 (1997).

[6] E. N. Glezer and E. Mazur, Appl. Phys. Lett. 71, 882 (1997).

[7] D. Homoelle, S. Wielandy, A. L. Gaeta, N. F. Borrelli, and C. Smith, Opt. Lett. 24, 1311 (1999).

[8] H.-B. Sun, Y. Xu, S. Juodkazis, K. Sun, M. Watanabe, S. Matsuo, H. Misawa, and J. Nishii, Opt. Lett. 26, 325 (2001).

[9] B. C. Stuart, M. D. Feit, A. M. Rubenchik, B.W. Shore, and M. D. Perry, Phys. Rev. Lett. 74, 2248 (1995).

[10] D. Du, X. Liu, G. Korn, J. Squier, and G. Mourou, Appl. Phys. Lett. 64, 3071 (1994).

[11] H.-B. Sun, S. Juodkazis, M. Watanabe, S. Matsuo, H. Misawa, and J. Nishii, J. Phys. Chem. B 104, 3450 (2000).

[12] J. W. Chan, T. Huser, S. Risbud, and D. M. Krol, Opt. Lett. 26, 1726 (2001).

[13] J. Qiu, P. G. Kazansky, J. Si, K. Miura, T. Mitsuyu, K. Hirao, and A. L. Gaeta, Appl. Phys. Lett. 77, 1940 (2000).

[14] L. Sudrie, M. Franco, B. Prade, and A. Mysyrowicz, Opt. Commun. 171, 279 (1999).

[15] J. D. Mills, P. G. Kazansky, E. Bricchi, and J. J. Baumberg, Appl. Phys. Lett. 81, 196 (2002).

[16] S. R. J. Brueck and D. J. Ehrlich, Phys. Rev. Lett. 48, 1678 (1982).

[17] D. Ashkenasi, H. Varel, A. Rosenfeld, S. Henz, J. Hermann, and E. E. B. Cambell, Appl. Phys. Lett. 72, 1442 (1998).

[18] D. H. Auston, K. P. Cheung, J. A. Valdmanis, and D. A. Kleinman, Phys. Rev. Lett. 53, 1555 (1984).

[19] C. B. Schaffer, A. Brodeur, and E. Mazur, Meas. Sci. Technol. 12, 1784 (2001).

[20] K. Tanimura, T. Tanaka, and N. Itoh, Phys. Rev. Lett. 51, 423 (1983). 